\title{
Genetic analysis for quality of frozen embryos produced by Holstein cattle donors in Canada
}

\author{
C. Jaton, ${ }^{*}{ }^{1}$ F. S. Schenkel, ${ }^{*}$ F. Malchiodi, ${ }^{*}$ M. Sargolzaei, ${ }^{*} \dagger$ C. A. Price, $\ddagger$ C. Baes, ${ }^{*}$ and F. Miglior ${ }^{*} \S$ \\ ${ }^{*}$ Centre for Genetic Improvement of Livestock, University of Guelph, Guelph, Ontario, Canada, N1G 2W1 \\ †The Semex Alliance, Guelph, Ontario, Canada, N1G $3 Z 2$ \\ łUniversité de Montréal, Faculté de Médecine Vétérinaire, St-Hyacinthe, Québec, Canada, J2S 2M2 \\ §Canadian Dairy Network, Guelph, Ontario, Canada, N1K 1E5
}

\begin{abstract}
The number of embryos produced by Holstein donors has been shown to be heritable, so it could be possible to genetically select for this trait to improve the efficiency of the assisted reproductive technology (ART) in dairy cattle. Another important parameter to consider for achieving good results from ART is embryo quality because embryos of good quality have more chance of producing live offspring. The possibility of using genetic selection for increasing the quality of embryo produced from ART has yet to be assessed. The objective of this study was, therefore, to perform a genetic analysis of embryo quality of Holstein donors in Canada using data recorded by Holstein Canada. The data set used was missing quality score data for embryos transferred fresh into a recipient, so the analyses were only performed for frozen embryos. With most traits in the Canadian dairy industry being evaluated with linear models, embryo quality was also evaluated with this class of models. However, considering the categorical nature of embryo quality, a threshold model was also evaluated. Embryo quality data were analyzed with either a univariate linear animal model or a univariate binomial threshold animal model. Genetic parameters estimated from the different models were comparable. A low heritability was found for the donor $(0.04 \pm<0.01)$ and the service sire $(0.02 \pm<0.01)$, but the repeatability estimate for the donor was higher (0.17), indicating that it was worthwhile to use a repeated records model. Overall, considering the low genetic parameters estimated, slow genetic progress is expected for the quality of frozen embryos produced by Canadian Holstein donors. Rank correlations were calculated between breeding values estimated from different models. High correlations were found between all models, indicating that no substantial re-ranking of the animals is expected from the
\end{abstract}

Received March 9, 2017.

Accepted May 18, 2017.

${ }^{1}$ Corresponding author: cjaton@uoguelph.ca different models. So, even though a threshold model is better suited for the analysis of categorical data, a linear model could be used for the analysis of embryo quality because it is less computationally demanding.

Key words: embryo quality, Holstein, genetic parameter, breeding value

\section{INTRODUCTION}

In dairy cattle, like in other species, the main goal of assisted reproductive technologies (ART) is to produce more live offspring from elite animals in a short period of time, aiming to accelerate the genetic gain of a population. To improve ART, 2 important parameters should be considered: the number of embryos produced and the quality of those embryos. In the short term, improving the technical aspects of ART could help produce more embryos of higher quality, but for a long-term improvement, genetic selection might be considered. In a previous study it was shown that it is possible to genetically select Holstein donors for increased embryo production (Jaton et al., 2016a). However, the possibility of genetic selection for improving embryo quality has yet to be assessed.

Embryos of higher quality usually have a higher chance of yielding a healthy offspring after being implanted in a recipient (Bó and Mapletoft, 2013). The birth of the offspring represents the ultimate assessment of embryo quality, but this implies a waiting period of at least 9 mo (Kanka et al., 2012). Therefore, other methods have been developed for evaluation of embryo quality to identify which embryos have a higher likelihood of getting a pregnancy (Van Soom et al., 2003). Nowadays, the most practical and noninvasive approach for choosing the best embryos for transfer is based on the morphology of the embryos (Van Soom et al., 2003; Farin et al., 2007; Kanka et al., 2012). In Canada and around the world, embryo quality is assessed visually by certified practitioners that use the classification system of the International Embryo Technology Society (IETS; Merton et al., 2003). To 
increase the uniformity across practitioners worldwide, IETS provides standardized coding systems that can help to assess the quality of the embryo at different stages of development (Bó and Mapletoft, 2013). Some of the factors that are considered for the morphological assessment of an embryo include color, developmental stage, size, compaction, and the presence of cellular debris (Van Soom et al., 2003; Bó and Mapletoft, 2013).

Large data sets of phenotypes measured at embryo recovery are required to perform a genetic analysis of embryo quality. Holstein Canada has such a data set, and the objective of the present study were therefore (1) to characterize the embryo quality data set, and (2) to estimate genetic parameters and EBV for embryo quality using different statistical models.

\section{MATERIALS AND METHODS}

\section{Data}

The data were provided by Holstein Canada and contained all viable embryos produced in Canada from 1980 to 2016, for a total of 1,334,414 records. This data set was complementary to the flushing data set analyzed in a previous study (Jaton et al., 2016b). Therefore, edits similar to Jaton et al. (2016b) were performed to include the same 150,971 superovulation and ovum pick-up and in vitro production of embryos (OPU-IVP) procedures. A total of 1,150,558 viable embryos were left after matching both data sets. These embryos were produced between January 1992 and January 2016 by 59,586 donors across Canada.

Around $36 \%$ of retained embryos were transferred fresh into a recipient and $64 \%$ were frozen after recovery. Key information was missing for the fresh embryos, namely the quality and the stage of development. Therefore, only frozen embryos were considered for further analysis. Moreover, additional edits were performed to only keep regular embryos, with complete information. Consequently, all embryos that were biopsied, divided, or produced by nuclear transfer were excluded from the data set. Veterinarians assessed the quality of the embryo with a score between 1 and 4, as suggested by IETS. Considering that embryos scored 4 for quality (n $=160$ ) were considered to be dead or degenerating, they were excluded from the data set. Three quality scores were considered: 1 (excellent), 2 (good), and 3 (poor). In addition, considering the low number of embryos of quality 3 ( $\mathrm{n}=4,932)$, these embryos were grouped with quality 2 embryos for some analyses. The stage of development of the embryo was scored on a linear 1 to 9 scale, as defined by IETS. A higher score corresponds to a more developed embryo. Embryos with a develop- mental stage between 2 (2- to 12-cell) and 8 (hatched blastocyst) were included, whereas stages 1 (unfertilized; $\mathrm{n}=253$ ) and 9 (expanded hatched blastocyst; $\mathrm{n}=$ 54) were excluded. At recovery, the embryos need to be washed with sterile medium and trypsin, which is especially important if the embryos are going to be exported (Farin et al., 2007). Nowadays, it is recommended to wash the embryos 10 times with sterile medium (Farin et al., 2007) and 2 times with trypsin for a total of 12 times washed. In the data set, information about the number of times the embryos were washed was known, but information about the washing solution used was not available. The number of times the embryos were washed was grouped into 4 classes: embryos that were washed less than 10 times, embryos that were washed 10 and 11 times, embryos that were washed 12 times, and embryos that were washed more than 12 times. The data set also contained information about the zona pellucida, which is a spherical layer that surrounds the embryo between the zygote and blastocyst stage (Van Soom et al., 2003; Farin et al., 2007). In order for embryos to be certified for export, the zona pellucida must be intact. Records were excluded if information about the number of times the embryo was washed or the zona pellucida were missing.

The other information available was the service sire used for fertilization of the oocyte, the year and month of embryo recovery, the clinic that recovered the embryo, the age of the donor at recovery, and the type of service, which indicates if embryos were produced using superovulation or OPU-IVP. If superovulation was used, more details were available about the person that performed the insemination, who could be the herd owner or an AI technician. Only clinics with at least 100 viable embryos and only embryos recovered between 1992 and 2015 were considered. The final data set consisted of 714,534 viable embryos produced by 44,584 donors, 3,140 sires (sire of the donor), and 2,255 service sires (sire of the embryo).

A pedigree file containing 221,215 animals was generated by tracing the pedigrees of the donors with records 7 generations back.

\section{Models}

In many countries, most traits considered for genetic selection of dairy cattle are continuous and normally distributed so that they are analyzed using a standard linear model (Meijering and Gianola, 1985). However, ordered categorical traits, such as embryo quality, do not follow a normal distribution, so linear models may not be optimal for genetic selection purposes of these traits (Gianola and Foulley, 1983; Harville and Mee, 
1984; Meijering and Gianola, 1985). Therefore, other models, which are theoretically better at describing the data, should be considered for the analysis of binomial or multinomial traits (Hoeschele, 1988; Eaglen et al., 2012). In a threshold model, it is usually assumed that the ordered categorical trait results from an underlying continuous normal distribution in which one or multiple thresholds create the observed distribution (Gianola, 1982; Gilmour et al., 1985; Van Tassell et al., 1998). This concept was first postulated by Wright (1934) who analyzed the number of toes of Guinea pigs, which is a binomial trait. Even though the threshold model is more appropriate for the analysis of ordered categorical data, it is computationally more demanding and complicated than the linear model (Meijering and Gianola, 1985). For a given ordered categorical trait, if the results from a threshold and a linear model are comparable, it may be computationally easier to use a linear model. Some studies have shown similar rankings of animals using either model (Meijering and Gianola, 1985; Mayer, 1995), whereas other studies have shown a small significant superiority of threshold models over linear models (Hoeschele, 1988).

Another way to analyze ordered categorical data is to use the scoring procedure proposed by Snell (1964), in which numerical scores for subjectively defined categories are determined assuming the underlying trait follows a logistic distribution (Tong et al., 1977; Gianola, 1982). This scoring procedure has been used for conformation and calving ease traits in cattle (Tong et al., 1977).

Embryo quality data were analyzed with a univariate linear animal model with either untransformed data or data transformed using the procedure of Snell (1964). A second series of analyses was performed considering only 2 quality scores (1: excellent; 2: good or poor). This second set of data was analyzed using either a univariate linear animal model or a univariate binomial threshold animal model. Therefore, 4 distinct analyses were performed:

1) Linear animal model with 3 quality scores (1: excellent; 2: good; 3: poor),

2) Linear animal model with transformed data using Snell procedure (1: excellent; 2: good; 3: poor),

3) Linear animal model with 2 quality scores (1: excellent; 2: good or poor), and

4) Binomial threshold animal model with 2 quality scores (1: excellent; 2: good or poor).

Data were analyzed in the DMU package (Madsen and Jensen, 2008) using either a univariate linear mixed model in the AI-REML procedure or a univariate binomial threshold mixed model via generalized linear mixed model (GLMM) using a probit link function (Tempelman, 1998).

The following linear mixed model was applied for embryo quality:

$$
y=\mathbf{X} \boldsymbol{\beta}+\mathbf{Z}_{\mathbf{d}} \mathbf{d}+\mathbf{Z}_{\mathrm{ss}} \mathbf{s s}+\mathbf{Z}_{\mathbf{p e}} \mathbf{p e}+\mathbf{e},
$$

where $y$ is the embryo quality; $\boldsymbol{\beta}$ is a vector of systematic fixed effects, including the effects of age (as linear and quadratic covariate) nested within service type, year-month of recovery, clinic-year of recovery, stage of development, number of times washed, and the zona intact; $\mathbf{d}$ is a vector of random animal additive genetic effects of the donor cow; ss is a vector of random animal additive genetic effects of the service sire; pe is a vector of random permanent environmental effects of the donor; $\mathbf{e}$ is a vector of random residuals; and $\mathbf{X}, \mathbf{Z}_{\mathbf{d}}$, $\mathbf{Z}_{\mathrm{ss}}$, and $\mathbf{Z}_{\mathrm{pe}}$ are the corresponding incidence matrices.

Random effects were assumed to be normally distributed with means equals to zero and covariance structure equal to

$$
\operatorname{Var}\left[\begin{array}{c}
d \\
s s \\
p e \\
e
\end{array}\right]=\left[\begin{array}{cccc}
\mathbf{A} \sigma_{d}^{2} & \mathbf{A} \sigma_{d, s s} & 0 & 0 \\
\mathbf{A} \sigma_{d, s s} & \mathbf{A} \sigma_{s s}^{2} & 0 & 0 \\
0 & 0 & \mathbf{I} \sigma_{p e}^{2} & 0 \\
0 & 0 & 0 & \mathbf{I} \sigma_{e}^{2}
\end{array}\right] \text {, }
$$

where $\sigma_{d}^{2}, \sigma_{s s}^{2}, \sigma_{p e}^{2}$, and $\sigma_{e}^{2}$ are donor, service sire, permanent environmental, and residual variances, respectively; and $\sigma_{d, s s}$ is the covariance between donor and service sire additive genetic effects. A and $\mathbf{I}$ are the additive genetic relationship and identity matrices, respectively. For the GLMM, the model specification was

$$
E(y \mid \mathbf{p})=\mathbf{p}=\Phi\left(\mathbf{X} \boldsymbol{\beta}+\mathbf{Z}_{\mathbf{d}} \mathbf{d}+\mathbf{Z}_{\mathbf{s s}} \mathbf{s s}+\mathbf{Z}_{\mathbf{p e}} \mathbf{p e}\right),
$$

where $E()$ is the expectation operator, $\mathbf{p}$ is a vector of probability of excellent quality score for each animal, $\Phi$ is the normal cumulative density function, and the other terms are as previously defined.

The fixed effects included in the models were tested for significance using the HPMIXED procedure of SAS (SAS Institute, 2013) accounting for repeated records. All effects tested were found highly significant $(P<$ $0.0001)$ by an $F$-test and were included in the model.

Genetic Parameters. For the linear model, heritability $\left(\mathrm{h}^{2}\right)$ and repeatability $(\mathrm{r})$ were calculated as follows for the donor $(d)$ : 


$$
\begin{gathered}
h_{d}^{2}=\sigma_{d}^{2} /\left(\sigma_{d}^{2}+2 \sigma_{d, s s}+\sigma_{s s}^{2}+\sigma_{p e}^{2}+\sigma_{e}^{2}\right), \\
r_{d}=\left(\sigma_{d}^{2}+\sigma_{p e}^{2}\right) /\left(\sigma_{d}^{2}+2 \sigma_{d, s s}+\sigma_{s s}^{2}+\sigma_{p e}^{2}+\sigma_{e}^{2}\right),
\end{gathered}
$$

and for the service sire $(s s)$ :

$$
h_{s s}^{2}=\sigma_{s s}^{2} /\left(\sigma_{d}^{2}+2 \sigma_{d, s s}+\sigma_{s s}^{2}+\sigma_{p e}^{2}+\sigma_{e}^{2}\right) .
$$

For a threshold model, the heritability is obtained on the underlying scale and is independent of the threshold value (Dempster and Lerner, 1950). For linear models the heritability depends on the frequencies of the categories in the population (Gianola, 1982). To compare the genetic parameters from both models, heritabilities from the binomial threshold model were transformed from the underlying $\left(h_{u n d}^{2}\right)$ to the observed $\left(h_{o b s}^{2}\right)$ scale using the equation proposed by Dempster and Lerner (1950):

$$
h_{\text {obs }}^{2}=h_{\text {und }}^{2} \times z_{\text {ord }}^{2} /[p \times(1-p)]
$$

where $p$ is the proportion of quality 1 embryos and $z_{\text {ord }}^{2}$ is the ordinate height of the normal distribution at the threshold point corresponding to $p$.

$\boldsymbol{E} \boldsymbol{B} \boldsymbol{V}$. Spearman rank correlations between sire estimated breeding values predicted from the 4 different models were calculated using the CORR procedure of SAS (SAS Institute Inc., 2013). Note that for the threshold model, the estimates from the underlying scale were used for the estimation of breeding values.

\section{RESULTS AND DISCUSSION}

\section{Descriptive Statistics}

As stated earlier, 714,534 viable embryos were included in the analysis. The distribution of embryo quality is presented in Table 1. The majority (86.2\%) of embryos were scored 1 (excellent), whereas 13.1 and $0.7 \%$ of embryos were scored 2 (good) or 3 (poor), respectively.

Table 1. Frozen embryo quality (Q1: excellent; Q2: good; Q3: poor) by clinic

\begin{tabular}{lcccc}
\hline Item & $\begin{array}{c}\text { No. of records } \\
\text { by clinic }\end{array}$ & Q1 (\%) & Q2 (\%) & Q3 (\%) \\
\hline All clinics & 714,534 & 86.2 & 13.1 & 0.7 \\
Mean & 6,937 & 86.4 & 12.6 & 1.0 \\
Minimum & 126 & 47.6 & 0.3 & 0.0 \\
Maximum & 77,446 & 99.7 & 52.3 & 7.3 \\
\hline
\end{tabular}

This distribution was expected because embryos of better quality are usually chosen to be frozen over embryos of lower quality.

Year and Month of Recovery. The distribution of records and the percentage of embryos for each quality score per year are presented in Figure 1a. The number of frozen embryos produced in Canada has increased over the years, but now seems to have reached a plateau at about 40,000 frozen embryos per year. The decreasing trend for 2015 only indicates that the data set is not yet complete for that year. Overall, embryo quality was stable over the years, with a slightly lower percentage of quality 1 embryos in years 1994 to 1996 . Similarly, Figure 1b shows that embryo quality was also constant over months, but we noticed a very slight decrease of excellent embryos in July and August, months in which the temperature in Canada is the highest. It has been reported that heat stress, which is well known for affecting dairy cattle fertility, can also negatively affect embryo quality (Sartori et al., 2010), which could explain the small decrease observed. Also, a decrease occurred in the number of records for summer months, which indicates that fewer frozen embryos were produced during these months. When all embryos were considered, even the ones implanted fresh, a similar decrease in the number of embryos produced during the summer months was observed.

Clinic. One hundred three clinics were included in the analyses, and the number of records per clinic was very different from one clinic to the other. The percentage of embryos in the different quality scores also varied greatly across clinics. The clinics had between 47.6 and $99.7 \%$ of excellent embryos, between 0.3 and $52.3 \%$ of good embryos and between 0 and $7.3 \%$ of poor embryos.

Service Type. Very few embryos from this data set were produced in vitro $(0.96 \%)$ as presented in Table 2. The percentage of excellent quality embryo was similar for all service type, but was slightly higher for embryo produced in vivo, for which insemination was performed by the herd owner. Van Soom et al. (2003) reported in vivo-derived cattle embryos to have a better quality than in vitro-produced embryos. This is also the case in our study. However, the facts that we have very few in vitro records and that we only have data for frozen embryos are limitations that need to be considered. Differences between in vivo and in vitro derived embryos are expected because the procedure can influence the morphology of the embryo (Van Soom et al., 2003). It was reported that for in vitro-produced embryos, the quality score is much more laboratory specific (Merton et al., 2003) than for embryos produced in vivo. Therefore, it is more difficult to directly compare the quality of embryos produced with different procedures. 
Table 2. Frozen embryo quality (Q1: excellent; Q2: good; Q3: poor) by service type, intact zona pellucida, and number of times the embryo was washed

\begin{tabular}{llrrrr}
\hline \multirow{2}{*}{ Fixed effect } & Level & $\begin{array}{r}\text { No. of } \\
\text { records }\end{array}$ & Q1 (\%) & Q2 (\%) & Q3 (\%) \\
\hline Service type & In vivo (herd owner) & 289,527 & 88.7 & 10.7 & 0.6 \\
& In vivo (AI technician) & 418,132 & 84.5 & 14.7 & 0.8 \\
Intact zona pellucida & In vitro & 6,875 & 84.5 & 15.4 & 0.1 \\
& Yes & 673,603 & 87.1 & 12.2 & 0.7 \\
Number of times the & No & 40,931 & 71.1 & 28.3 & 0.6 \\
embryo was washed & 1 to 9 & 38,292 & 83.46 & 15.23 & 1.31 \\
& 10 or 11 & 158,455 & 86.98 & 11.98 & 1.04 \\
& 12 & 506,918 & 86.05 & 13.42 & 0.54 \\
& 13 or more & 10,869 & 90.45 & 9.01 & 0.54 \\
\hline
\end{tabular}

Age of the Donor. As presented in Figure 2, the percentage of excellent quality embryos was slightly higher for younger donors, whereas the percentage of poor quality embryos was slightly higher for older donors. Figure 3 shows the distribution of excellent and good quality embryos by the age of the donor within

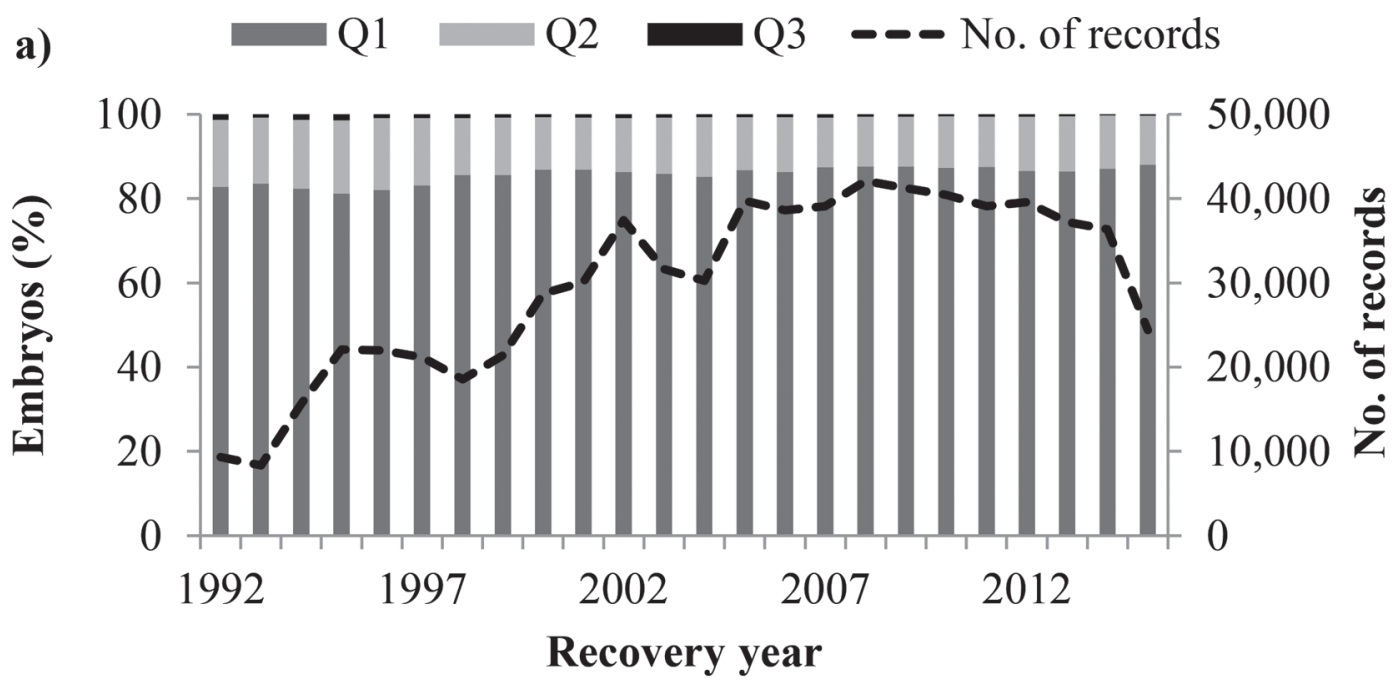

b) $\quad$ Q1 $\quad$ Q2 $Q 3 \quad--$ No. of records

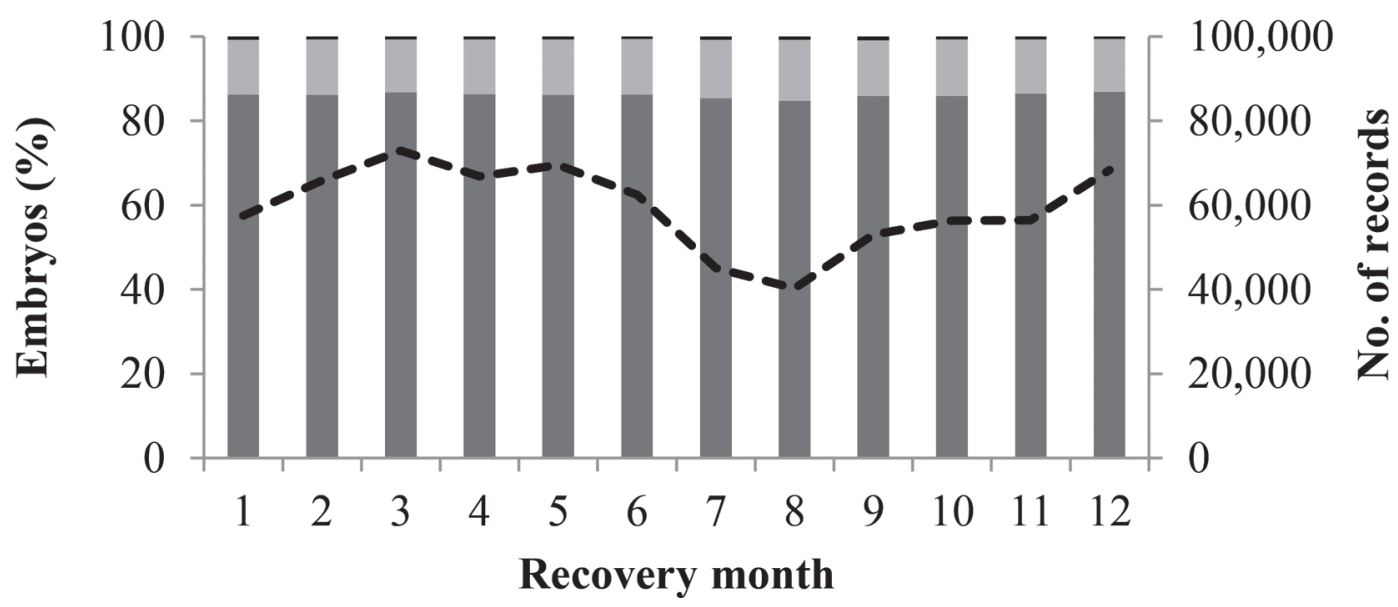

Figure 1. Frozen embryo quality (Q1: excellent; Q2: good; Q3: poor) by (a) year of recovery and (b) month of recovery. 


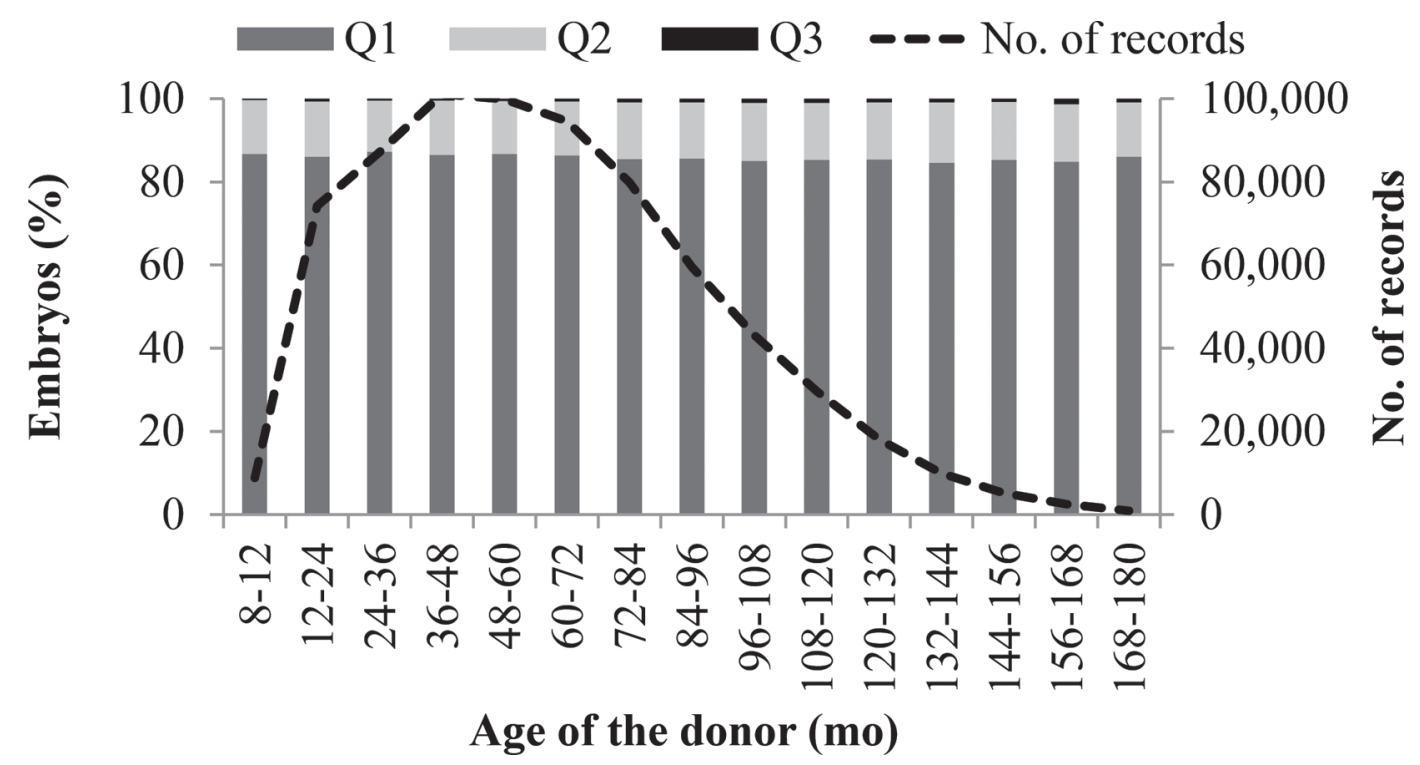

Figure 2. Frozen embryo quality (Q1: excellent; Q2: good; Q3: poor) by age of the donor.

the service type. For embryos produced in vivo, a slight decrease was observed of excellent quality embryos with the aging of the donor. For in vitro-produced embryos, no clear trend was observed for embryo quality with the age of the donor.

Stage. Figure 4 shows the variation of embryo quality considering the stage of development of the embryo at recovery. The percentage of embryos of excellent quality was highest for stages 6 and 7 . Most of the frozen embryos were of stages 4 (morula), 5 (early blastocyst), and 6 (blastocyst). For superovulation, the donors are synchronized so that most of the embryos will be at those stages at recovery, because they usually yield the highest pregnancy rates (Bó and Mapletoft, 2013). However, embryos are produced from follicles that are of different sizes and stages when they are recruited during the follicular wave (Merton et al., 2003), so it is expected to recover embryos at different stages

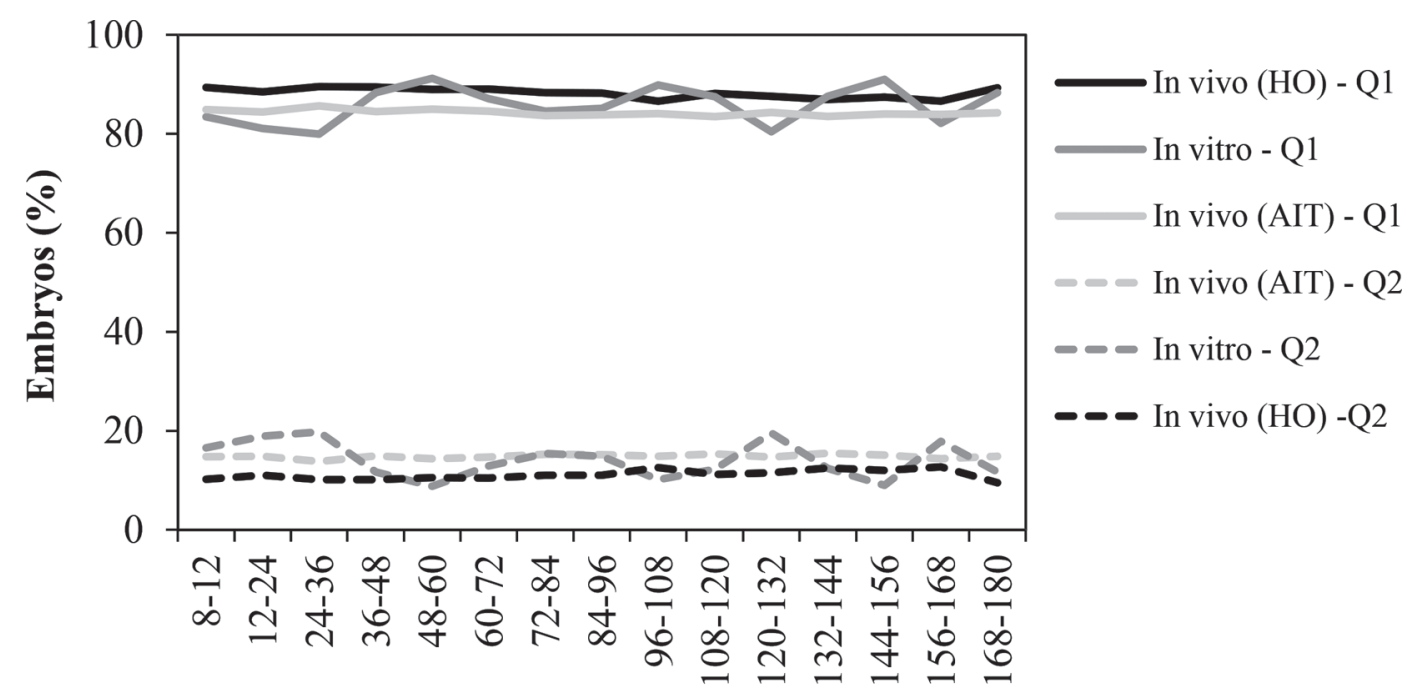

Age of the donor (mo)

Figure 3. Frozen embryo quality (Q1: excellent; Q2: good) by age of the donor within service type (HO = herd owner; AIT = artificial insemination technician). 


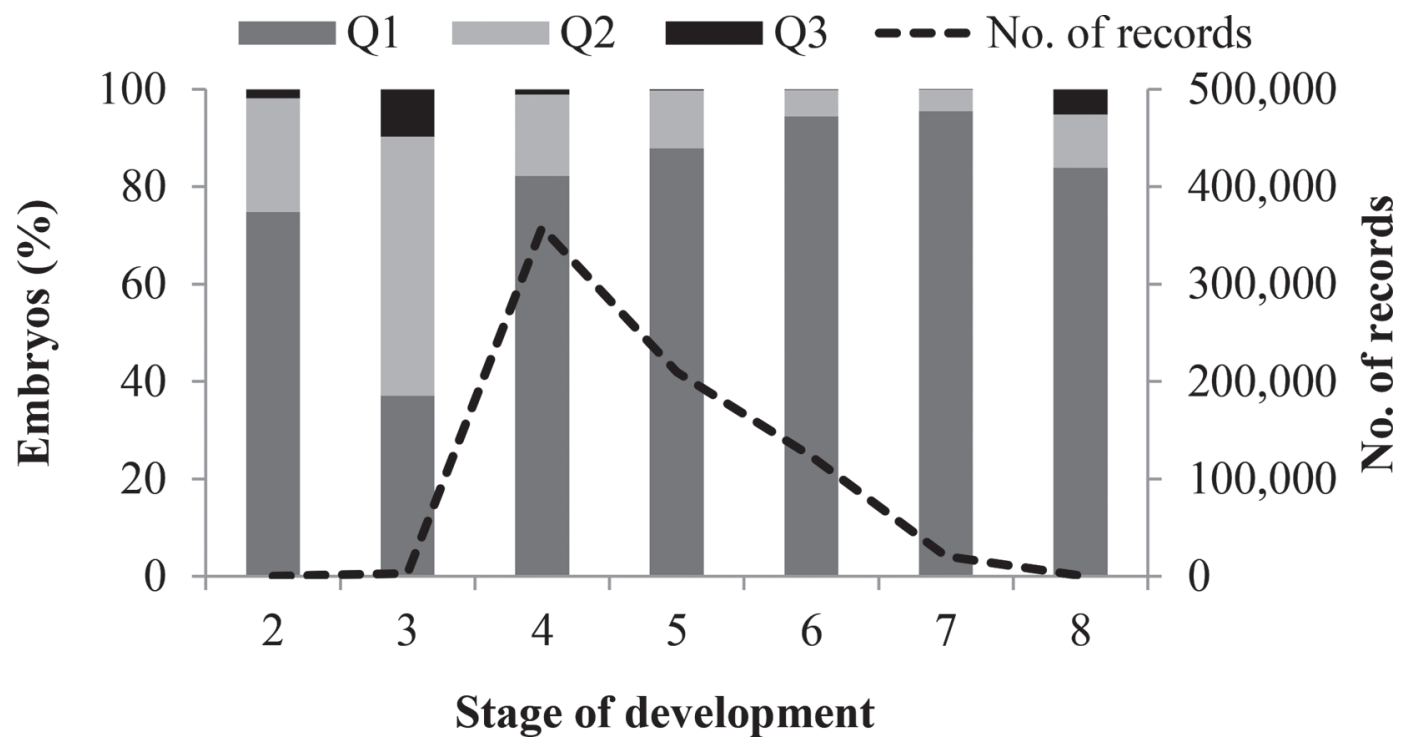

Figure 4. Frozen embryo quality (Q1: excellent; Q2: good; Q3: poor) by stage of development.

(Bó and Mapletoft, 2013). For OPU-IVP, more homogeneous embryos are expected if repetitive sessions are performed on a donor (Merton et al., 2003).

Zona Pellucida Intact. The majority of the frozen embryos (94.3\%) included in this study had an intact zona pellucida. The embryos that did not have an intact zona pellucida were on average of lower quality (Table 2).

Number of Times Washed. As described earlier, it is recommended to wash the embryos 12 times and this is in accordance with our data set, given that $70.9 \%$ of the embryos were washed 12 times. The percentage of embryos of excellent quality increased with the number of times the embryos were washed (Table 2).

\section{Genetic Parameters}

Genetic parameters estimated for embryo quality are presented in Table 3. Similar results were obtained for all models for this data set. The heritability estimates for the donor were low at $0.042 \pm 0.004,0.042 \pm 0.004$, and $0.038 \pm 0.004$ for linear models 1,2 , and 3, respectively. For model 4 (threshold) similar heritability of the donor was found (0.034) after transformation from the underlying to the observed scale. These low heritability estimates for the donor indicate that even though it could still be possible to genetically select for increased embryo quality, rate of genetic progress for this trait would be slow. The repeatability of the donor was higher at around 0.17 for all linear models. This indicates that it is worthwhile to use a repeated records model, but it also suggests that a donor is not expected to produce embryos of similar quality over subsequent superovulation or OPU-IVP sessions. For the service sires, the heritabilities were very low at $0.016 \pm 0.001$, $0.018 \pm 0.002$, and $0.020 \pm 0.002$ for models 1,2 , and 3 , respectively. For the threshold model, heritability of the service sire on the observed scale was also very low (0.010), implying that the service sire has a limited genetic effect on the embryo quality. To our knowledge, no other studies have estimated genetic parameters for embryo quality. However, one study estimated the heritability of the percentage of excellent quality of oocytes recovered by OPU session. Using a sire model they found a heritability of $0.09 \pm 0.02$, which is slightly higher, but in the same range as our estimate for the donor (Merton et al., 2009).

\section{$E B V$}

Breeding values were estimated for all models for both the donors $(\mathrm{n}=44,584)$ and the sires $(\mathrm{n}=3,140)$. The Spearman rank correlations between the EBV estimated using different models for the all sires and for sires with at least 20 daughters with records are presented in Table 4 . In all cases, the highest correlations were between models 1 and 2, which can be explained by the fact that 3 quality scores were used for both models and that they were both linear. Similarly, for the threshold model, the highest correlation was found with model 3, in which there were also 2 quality scores. Correlations between all linear models were slightly higher than the correlations between the threshold and the linear models. 


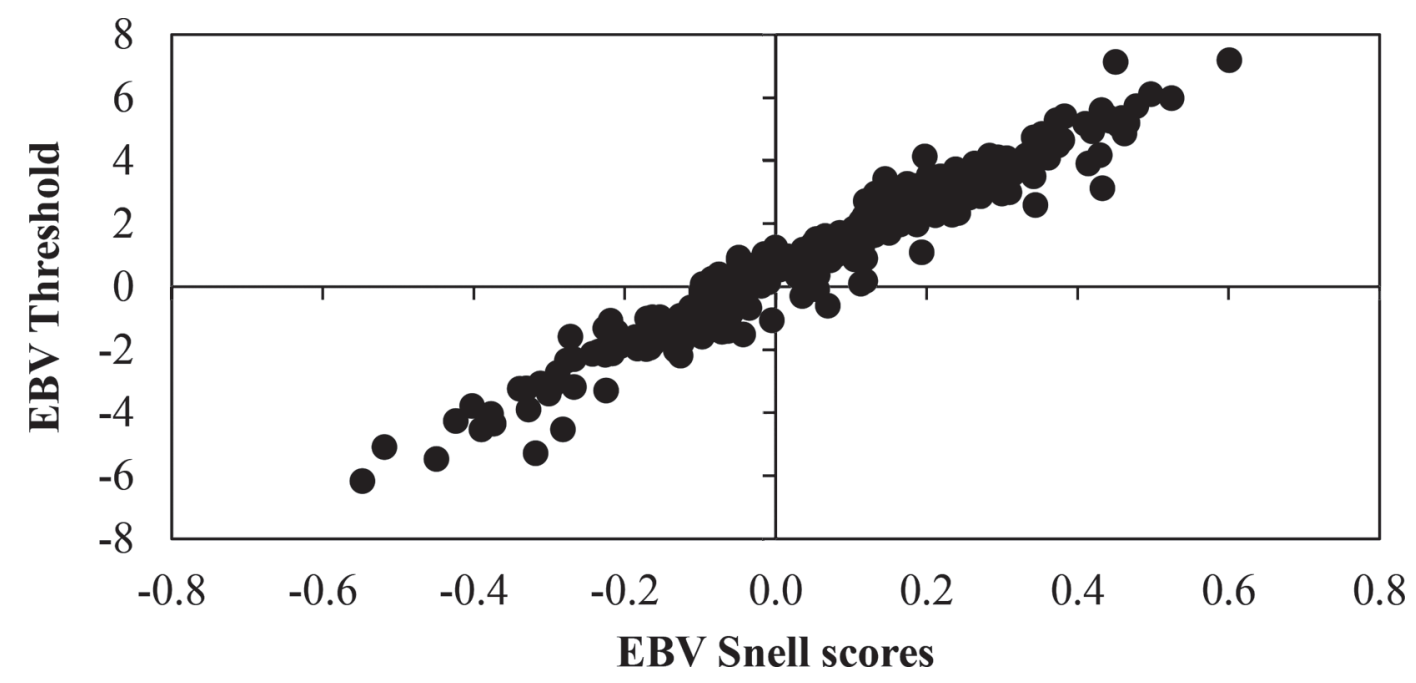

Figure 5. Estimated breeding values from models 2 (linear Snell scores) and 4 (threshold) for sires with at least 20 daughters $(\mathrm{n}=272$ ).

Higher rank correlations were found when sires with at least 20 daughters were considered. This was expected considering that these sires have more reliable EBV. Figure 5 shows the distribution of EBV obtained from models 2 and 4 for sires with at least 20 daughters. This figure indicates that substantial re-ranking of the animals is not expected using the different models for quality of frozen embryos.

Figure 6 shows the percentage of excellent quality embryos per donor per sire and the EBV of the sire as obtained in model 1. Overall, this figure indicates that sires with high EBV for embryo quality are more likely to have daughters that produce more excellent quality embryos than daughters of sires with lower EBV for this trait. Therefore, selecting sires with high genetic potential for embryo quality will likely results in more high quality embryos being produced by their daughters.

The linear model with 2 quality scores had the highest EBV correlation with the threshold model and required no additional data transformation. Therefore, it should be considered for the genetic evaluation of embryo quality. A threshold model is theoretically more appropriate for the analysis of categorical data (Gianola, 1982). However, considering the results obtained in this study and the fact that linear models are less complicated and less computationally demanding than threshold models (Meijering and Gianola, 1985), a linear model could be used for the analysis of embryo quality.

\section{Final Remarks}

The availability of quality data for frozen embryos only is a limitation of this study. Usually, only the best quality embryos are frozen, so the data set does not represent the full population of viable embryos produced in Canada. However, the large size of the data set enabled us to estimate valuable genetic parameters for the quality of frozen embryos. It would be worth-

Table 3. Genetic parameter ${ }^{1}$ estimates (SE in parentheses) for embryo quality using linear or binomial threshold models

\begin{tabular}{|c|c|c|c|}
\hline Model & $h_{d}^{2}$ & $r_{d}$ & $h_{s s}^{2}$ \\
\hline $\begin{array}{l}\text { (1) Linear (3 quality scores) } \\
\text { (2) Linear Snell } \\
\text { (3) Linear (2 quality scores) } \\
\text { (4) Threshold } \\
\text { (4) Threshold }\end{array}$ & $\begin{array}{l}0.042(0.004) \\
0.042(0.004) \\
0.038(0.004) \\
0.081(0.007) \\
0.034\end{array}$ & $\begin{array}{l}0.172 \\
0.172 \\
0.170 \\
0.286 \\
-\end{array}$ & $\begin{array}{l}0.016(0.001) \\
0.018(0.002) \\
0.020(0.002) \\
0.024(0.002) \\
0.010\end{array}$ \\
\hline
\end{tabular}

${ }^{1} h_{d}^{2}$ is the heritability of the donor, $r_{d}$ is the repeatability of the donor, and $h_{s s}^{2}$ is the heritability of the service sire.

${ }^{2}$ Observed scale.

${ }^{3}$ Underlying scale.

${ }^{4}$ Observed scale transformed from the underlying scale. 
Table 4. Spearman rank correlations of breeding values estimated using different models for all sires $(\mathrm{n}=$ $3,140$; above diagonal $)$ and for sires with at least 20 daughters $(\mathrm{n}=272$; below diagonal $)$

\begin{tabular}{lcccc}
\hline Model & $\begin{array}{c}\text { (1) Linear } \\
\text { (3 quality scores) }\end{array}$ & $\begin{array}{c}\text { (2) Linear } \\
\text { Snell scores }\end{array}$ & $\begin{array}{c}\text { (3) Linear } \\
\text { (2 quality scores) }\end{array}$ & (4) Threshold \\
\hline (1) Linear (3 quality scores) & 0.999 & 0.999 & 0.987 & 0.964 \\
(2) Linear Snell & 0.989 & 0.995 & 0.994 & 0.970 \\
(3) Linear (2 quality scores) & 0.967 & 0.973 & 0.981 & 0.974 \\
(4) Threshold & & & \\
\hline
\end{tabular}

while to run all models with data of embryos that were frozen and that were transferred fresh to see if similar genetic parameters would be estimated and if similar conclusions would be reached about the ideal model to use.

Using genetic evaluation for embryo quality could be very useful for the dairy industry, especially if it was combined with the evaluation for number of embryos. Having an index that combines those 2 sources of information could help breeders select animals that would produce more embryos of higher quality and could help to improve the efficiency of embryo production programs. The evaluation for embryo quality information could also be beneficial for research programs aiming to identify genes that have an effect on the quality of embryos, which, in turn, could help accelerate genetic progress for production of high-quality embryos.

\section{CONCLUSIONS}

The results obtained in this study provide evidence of potential to genetically select for embryo quality in the Canadian Holstein population, but considering the low heritability of this trait, the genetic progress would be slow using traditional genetic evaluation. Using different models yielded similar genetic parameters for both the donor and the service sire. Considering the high EBV rank correlations estimated between the linear

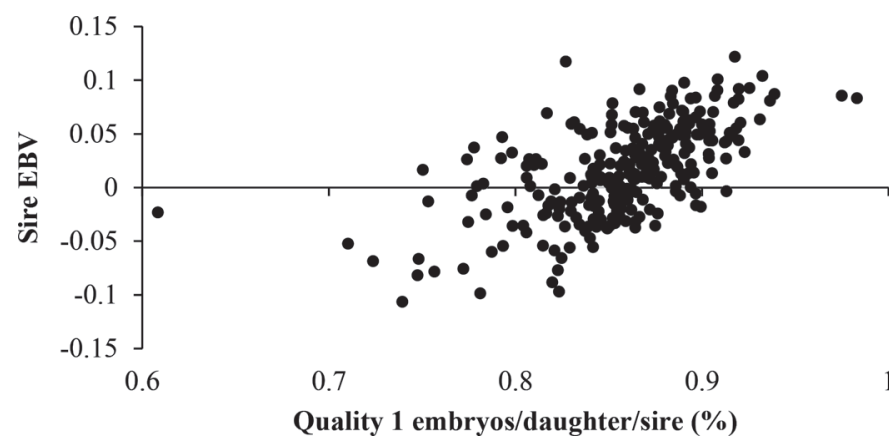

Figure 6. Percentage of excellent quality (Q1) embryos per donor per sire by sire EBV from model 1 (linear 3 quality scores) for sires with at least 20 daughters $(\mathrm{n}=272)$. models and the threshold model, a simple linear model seems appropriate for the genetic evaluation of quality of frozen embryos. Even though threshold models are theoretically recommended for the evaluation of categorical traits, they are more computationally demanding so it would be more efficient to use a linear model.

\section{ACKNOWLEDGMENTS}

The authors are grateful to Holstein Canada for providing the data. This study was funded by the Centre d'insémination artificielle du Québec (St-Hyacinthe, Quebec, Canada), The Semex Alliance (Guelph, Ontario, Canada), the DairyGen Council of Canadian Dairy Network (Guelph, Ontario, Canada), and the Natural Sciences and Engineering Research Council of Canada (Ottawa, Ontario, Canada).

\section{REFERENCES}

Bó, G. A., and R. J. Mapletoft. 2013. Evaluation and classification of bovine embryos. Anim. Reprod. Sci. 10:344-348

Dempster, E. R., and I. M. Lerner. 1950. Heritability of threshold characters. Genetics 35:212-236.

Eaglen, S. A. E., M. P. Coffey, J. A. Woolliams, and E. Wall. 2012. Evaluating alternate models to estimate genetic parameters of calving traits in United Kingdom Holstein-Friesian dairy cattle. Genet. Sel. Evol. 44:23.

Farin, P. W., K. Moore, and M. Drost. 2007. Assisted reproductive technologies in cattle. Pages 496-508 in Large Animal Theriogenology. Saunders Elsevier, Philadelphia, PA.

Gianola, D. 1982. Theory and analysis of threshold characters. J. Anim. Sci. 54:1079.

Gianola, D., and J. Foulley. 1983. Sire evaluation for ordered categorical data with a threshold model. Genet. Sel. Evol. 15:201-224.

Gilmour, A. R., R. D. Anderson, and A. L. Rae. 1985. The analysis of binomial data by a generalized linear mixed model. Biometrika $72: 593$.

Harville, D. A., and R. W. Mee. 1984. A mixed-model procedure for analyzing ordered categorical data. Biometrics 40:393.

Hoeschele, I. 1988. Comparison of "maximum a-posteriori estimation" and "quasi best linear unbiased prediction" with threshold characters. J. Anim. Breed. Genet. 105:337-361.

Jaton, C., A. Koeck, M. Sargolzaei, F. Malchiodi, C. A. Price, F. S. Schenkel, and F. Miglior. 2016a. Genetic analysis of superovulatory response of Holstein cows in Canada. J. Dairy Sci. 99:3612-3623.

Jaton, C., A. Koeck, M. Sargolzaei, C. A. Price, C. Baes, F. S. Schenkel, and F. Miglior. 2016b. Short communication: Genetic correlations between number of embryos produced using in vivo and in vitro techniques in heifer and cow donors. J. Dairy Sci. 99:82228226 . 
Kanka, J., L. Nemcova, T. Toralova, K. Vodickova-Kepkova, P. Vodicka, M. Jeseta, and M. Machatkova. 2012. Association of the transcription profile of bovine oocytes and embryos with developmental potential. Spec. Issue 3rd Embryo Genomics Meet. 134, 29-35.

Madsen, P., and J. Jensen. 2008. An user's guide to DMU. A package for analyzing multivariate mixed models. Version 6 , release 4.7. Danish Institute of Agricultural Sciences, Tjele, Denmark.

Mayer, M. 1995. Inequality of maximum a posteriori estimators with equivalent sire and animal models for threshold traits. Genet. Sel. Evol. 27:1-13.

Meijering, A., and D. Gianola. 1985. Linear versus nonlinear methods of sire evaluation for categorical traits: a simulation study. Genet. Sel. Evol. 17:115.

Merton, J. S., B. Ask, D. C. Onkundi, E. Mullaart, B. Colenbrander, and M. Nielen. 2009. Genetic parameters for oocyte number and embryo production within a bovine ovum pick-up-in vitro production embryo-production program. Theriogenology 72:885-893.

Merton, J. S., A. P. de Roos, E. Mullaart, L. de Ruigh, L. Kaal, P. L. Vos, and S. J. Dieleman. 2003. Factors affecting oocyte quality and quantity in commercial application of embryo technologies in the cattle breeding industry. Theriogenology 59:651-674.
Sartori, R., M. R. Bastos, and M. C. Wiltbank. 2010. Factors affecting fertilisation and early embryo quality in single- and superovulated dairy cattle. Reprod. Fertil. Dev. 22:151.

SAS Institute. 2013. SAS/STAT ${ }^{\circledR} 13.1$ User's Guide. SAS Institute Inc., Cary, NC.

Snell, E. J. 1964. A scaling procedure for ordered categorical data. Biometrics 20:592.

Tempelman, R. J. 1998. Generalized linear mixed models in dairy cattle breeding. J. Dairy Sci. 81:1428-1444.

Tong, A. K. W., J. W. Wilton, and L. R. Schaeffer. 1977. Application of a scoring procedure and transformations to dairy type classification and beef ease of calving categorical data. Can. J. Anim. Sci. $57: 1-5$.

Van Soom, A., B. Mateusen, J. Leroy, and A. de Kruif. 2003. Assessment of mammalian embryo quality: What can we learn from embryo morphology? Reprod. Biomed. Online 7:664-670.

Van Tassell, C. P., L. D. Van Vleck, and K. E. Gregory. 1998. Bayesian analysis of twinning and ovulation rates using a multiple-trait threshold model and Gibbs sampling. J. Anim. Sci. 76:2048-2061.

Wright, S. 1934. An analysis of variability in number of digits in an inbred strain of Guinea pigs. Genetics 19:506-536. 